\title{
The Bladder EpiCheck Test as a Non-Invasive Tool Based on the Identification of DNA Methylation in Bladder Cancer Cells in the Urine: A Review of Published Evidence
}

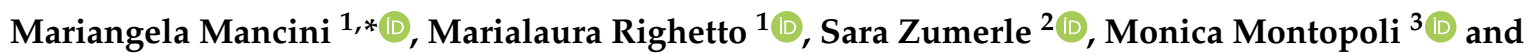 \\ Filiberto Zattoni ${ }^{1}$ \\ 1 Department of Surgical, Oncological and Gastroenterological Science, Urological Clinic, University of Padua, \\ 35128 Padua, Italy; marialaura.righetto@gmail.com (M.R.); filiberto.zattoni@unipd.it (F.Z.) \\ 2 Department of Medicine, Veneto Institute of Molecular Medicine, University of Padua, 35128 Padua, Italy; \\ sara@zumerle.net \\ 3 Department of Pharmaceutical and Pharmacological Sciences, University of Padua, 35128 Padua, Italy; \\ monica.montopoli@unipd.it \\ * Correspondence: mariangela.mancini@unipd.it
}

Received: 31 July 2020; Accepted: 2 September 2020; Published: 8 September 2020

\begin{abstract}
Recently, there has been a great effort to develop tests based on non-invasive urinary biomarkers (NMIBCs). These tests are based on the fact that NMIBCs are heterogeneous at the molecular level and can be divided into different molecular groups useful to predict prognosis and response to treatment. The assessment of epigenetic alterations, such as DNA methylation, represents a promising cancer biomarker. DNA methylation is an epigenetic modification that affects gene expression without modifying the DNA sequence. Several studies have highlighted the presence of methylated loci in the context of bladder cancer, indicating its potential application as a diagnostic and prognostic biomarker. One of the novel assays based on a DNA methylation profile, the Bladder EpiCheck, analyzes DNA from spontaneous urine, detecting disease-specific DNA methylation patterns in bladder cancer patients. This test, due to its non-invasive nature and highly promising performance could, in future, become an invaluable tool in the follow-up of bladder cancer patients. Potential new applications could include diagnosis and surveillance of upper-tract disease, for the replacement of invasive testing and ureteroscopy.
\end{abstract}

Keywords: bladder cancer; DNA methylation; biomarkers

\section{Introduction}

\subsection{Background}

Non-muscle invasive bladder cancer (NMIBC) has high recurrence rates up to $60 \%$ within the first year of diagnosis [1]. Most patients will experience numerous recurrences during follow-up, while some others will experience progression to a muscle-invasive disease. The factors associated with the recurrence of NMIBC are grade (low-grade or high-grade disease), number and size of tumors, number of previous recurrences, tumor stage, and presence of concurrent carcinoma in situ [2]. Moreover, NMIBC is a dangerous chronic disease, leading to a substantial burden on patients and healthcare systems [3]. Surveillance of NMIBC to early-identify recurrence, progression, or localization in different areas of the urinary tract is crucial and is currently based on repeated cystoscopies and urine cytologies [1]. Both of these exams present some issues: cystoscopy is an invasive and expensive 
procedure that could potentially lead to life-threating morbidities; urine cytology, on the other hand, has low sensitivity, low diagnostic accuracy and is operator-dependent, despite being a less expensive exam and having a high-specificity for high-grade NMIBC.

Recently, therefore, there has been a great effort to develop tests based on non-invasive urinary biomarkers $[4,5]$. These tests are based on the fact that NMIBCs are heterogeneous at the molecular level and can be divided into different molecular groups useful to predict prognosis and response to treatment [6]. The assessment of epigenetic alterations, such as DNA methylation, represents a promising cancer biomarker. DNA methylation is an epigenetic modification that affects gene expression without modifying the DNA sequence. Several studies have highlighted the presence of methylated loci in the context of bladder cancer, indicating its potential application as a diagnostic and prognostic biomarker $[7,8]$. One of the novel assays based on a DNA methylation profile, the Bladder EpiCheck (BE), analyzes DNA from spontaneous urine, thereby detecting disease-specific DNA methylation patterns in bladder cancer patients.

\subsection{Accuracy of a Diagnostic Test}

The ideal urinary biomarker to use in clinical practice should have good cost-benefits and good sensitivity and specificity. Moreover, it should be simple and quick to interpret, with a good diagnostic accuracy and inexpensive. The urinary markers developed so far do not have high specificity and sensitivity, and they have a lot of false positives and false negatives, as compared to a reference threshold. Consequently, these markers increasingly have a reduced positive predictive value (PPV) and negative predictive value (NPV), the majority having sensitivity and specificity ranging between $50-80 \%$ [9].

We need a test with high NPV for all non-muscle invasive bladder cancers (NMIBCs), especially for high-grade NMIBCs. NPV is a point of reference because it could avoid unnecessary cystoscopies.

\subsection{Bladder EpiCheck Test}

The Bladder EpiCheck ${ }^{\mathrm{TM}}$ (Nucleix, Rehovot, Israel) test is a non-invasive assay recently developed for the surveillance of bladder cancer recurrence. The analysis is based on the detection of the DNA methylation status of 15 genomic loci which are associated with a high prevalence of DNA methylation in bladder cancer cells.

Ten milliliters of urine represent the minimal amount of urine to perform the assay reliably. Genomic DNA is extracted from cells obtained by urine centrifugation and digested using a methylation-sensitive restriction enzyme which cleaves DNA at specific sites only in the absence of methylation. The methylation status of the 15 biomarkers is then assessed by real-time polymerase chain reaction (PCR) and analyzed using a specific software, thus providing a numerical value called an EpiScore ${ }^{\mathrm{TM}}$. Samples with an EpiScore ${ }^{\mathrm{TM}}$ superior to 60, on a 0-100 range, are considered positive for bladder cancer.

\section{Evidence Acquisition}

\subsection{Search Strategies, Selection of Studies, and Data Extraction}

This is a narrative review. Databases including MEDLINE, Embase and the Cochrane Library were systematically searched. We used different combinations of the keywords "non-muscle-invasive bladder cancer", "urinary biomarker", "Bladder EpiCheck", and "DNA methylation test", and we included results up to 31 May 2020. No language restriction was applied. All studies describing the application of the Bladder EpiCheck test (BE) to patients with NMIBC were included, without exclusion criteria. We excluded from this review articles about $B E$ for the surveillance of the upper urinary tract urothelial carcinoma. Full-text articles and non-full-text articles only (i.e., conference abstracts) published on BE were included in this review. The authors screened articles independently. 


\subsection{Types of Participants Included}

Concerning all studies, the study population was limited to patients who were in surveillance for histologically proven NMIBC of any grade (carcinoma in situ - CIS, non-invasive papillary carcinoma $\mathrm{Ta}$, carcinoma that invades subepithelial connective tissue - T1; no regional lymph node metastasis clinically identified - cN0, no distant metastasis clinically identified - cM0) according to the TNM (tumor - nodes - metastasis) staging system (all versions). No gender limit was considered. Two studies [10,11] included only patients with NMIBC resected within the previous 12 months.

Data from patients with a first diagnosis of bladder cancer, patients with muscle-invasive bladder cancer, and patients with non-localized disease (with clinically positive regional lymph nodes or distant metastasis - $\mathrm{cN}+, \mathrm{cM}+$ ) were excluded in all studies.

Patients who were having adjuvant intravesical therapy and surveillance cystoscopies at 3-6-month intervals also were included in the studies.

\subsection{Types of Intervention and Outcome Measures Included}

BE was performed on patient urine samples collected before standard-of-care cystoscopy.

The primary outcomes were the sensitivity and specificity of BE during surveillance for NMIBC. Both sensitivity and specificity of the BE were compared to the cystoscopy, cytology, and histology results for a reference standard. The secondary outcome was the EpiScore continuous measure for each patient.

\subsection{Data Analysis}

Regarding each paper, we collected information on study design, inclusion and exclusion criteria, patient characteristics, and outcome measures. We used descriptive statistics to summarize baseline data and to plan a narrative synthesis of the data.

\section{Evidence Synthesis}

\subsection{Quantity of Evidence Identified and Characteristics of the Studies Included}

Overall, 11 records were identified through database searching, and eight were screened after removal of duplicates and of a study on upper tract urothelial carcinoma surveillance. Considering these, five articles were eligible for full-text screening, and three conference abstracts were assessed for eligibility.

Finally, eight studies recruiting a total of 1993 patients met the inclusion criteria. All the studies were prospective, blinded, single-arm cohort studies, with five regarding multicentric tumors [10-14] and three regarding monocentric tumors [15-17].

One study was a secondary external independent analysis of the results of a previous one [11], a second study was an updated version of a previous one, with additional patients [14], and a third study was a comparative study on two types of Real-Time Polymerase Chain Reaction-Based (RT-PCR) urinary markers, based on the population analyzed in a previous study [13].

\subsection{Reference Standard Definition and Follow-Ups}

Regarding all studies, the sensitivity and specificity of the BE were compared to a prespecified reference standard, defined as the result of cystoscopy, cytology, and/or histology. The definition of the reference standard and, consequently, of its result, is a key point in the evaluation of the positivity or negativity of the BE. Concerning the case of a positive cystoscopy (suspicious for recurrence), histology was performed, either an endoscopic cold-cup biopsy or a transurethral resection (TURB). Regarding the case of a positive/suspicious wash cytology, subsequent endoscopic mapping biopsies were planned. 
To define a 'negative' patient, the white-light cystoscopy, wash cytology, and histology must be negative. Particularly, for cytological specimen classification, all studies used the Paris System for Reporting Urinary Cytology. During three of the studies $[10,11,17]$ the wash cytology was considered 'negative' when the result was 'negative for high-grade urothelial carcinoma (HGUC)' and 'atypical urothelial cells', whereas 'suspicious for HGUC', 'HGUC', and 'low-grade (LG) intraepithelial neoplasia' were clustered as 'positive'. Concerning the case of positive spontaneous or wash cytology with negative cystoscopy and/or histology, the reference standard was evaluated at the subsequent follow-up cystoscopy with multiple random biopsies or targeted biopsies on a suspicious area [17]. Concurrently, in the study by Pierconti et al. [16], patients with a cytological diagnosis of 'atypical urothelial cells' also were considered 'positive' and underwent cystoscopy within three months, with multiple random biopsies in the case of a negative cystoscopy.

Notably, Witjes at al., [10] used more rigid parameters in the reference standard definition, considering the pathology specimen as the key determinant. When the patient had a positive pathology, the sample was considered 'positive'; when the pathology was negative, but the cytology was positive, the sample was considered inconclusive, and excluded from the final analysis. Similarly, samples with positive or equivocal cystoscopic or cytologic results without a subsequent confirmatory pathology also were classified as inconclusive and excluded from the final analysis.

Patient follow-ups after BE varied from six months [17] to 12 months [11-17]. Follow-up data was not available from one study [10].

\subsection{Statistical Methods}

All studies conducted a sensitivity analysis to check the performance of BE in the detection of low and high-grade bladder cancers. The discrimination of $\mathrm{BE}$ was assessed graphically with the receiver operating characteristic (ROC) curve and the area under the curve (AUC), an immediate measure for the EpiScore continuous variable. Additional statistics evaluating the accuracy of the BE included the positive and negative predictive value (PPV and NPV, respectively), the positive and negative likelihood ratio (PLR and NLR, respectively), and the receiver-operating curve (ROC). The area under the ROC curve (AUC) was a summary measure for the EpiScore continuous value.

Unlike the other studies, D'Andrea et al. [11] evaluated the association between the BE result and bladder cancer recurrence by generating two multivariate nomograms for the prediction of the presence of any-grade and high-grade bladder cancer, respectively. Moreover, the authors performed a decision-curve analysis (DCA) to evaluate the utility of BE on decision-making in routine clinical practice.

\subsection{Results: Sensitivity, Specificity, and Predictive Values Analysis}

The validation study by Wasserstrom et al. [15] applied BE on a sample population of 222 patients. Histological diagnosis of bladder cancer was possible for 40 patients (18\%), and BE correctly identified 36 malignancies, with an overall sensitivity of $90 \%$. Notably, BE sensitivity changed based on staging and grading, with a higher sensitivity in higher stages $(81 \%, 100 \%, 100 \%, 91 \%$, in Ta, T1, T2 (tumor invading muscularis) and CIS, respectively), and in higher grades ( $84 \%$ in low-grade, and $95 \%$ in high-grade tumors). The overall BE specificity was $83 \%$, correctly diagnosing 151/182 cancer-free patients, and the NPV was $97 \%$.

Conversely, considering wash cytology as the reference standard, BE showed a greater sensitivity ( $90 \%$ versus $38 \%$, in low and high-grade tumors), but a lower specificity ( $83 \%$ versus $96 \%$ ).

This means that BE detected all cancers that also were detected by cytology, and that all cancers missed by BE also were missed by cytology. Finally, the authors highlighted that high histological grading was significantly associated with a higher mean EpiScore score, than was low grading (85 versus 69 , respectively; $p=0.006$ ).

Witjes et al. [10] tested BE on 440 patients, performing statistical analysis on 353 of them ( 87 subjects excluded due to inconclusive diagnosis according to the reference standard, no BE results, or both). 
Forty-four out of three hundred and fifty-three patients (12.5\%) were 'positive' according to the reference standard.

The overall sensitivity was $68 \%$ (30/44) and the overall specificity was $88 \%(272 / 309)$. The test performance did not vary with age, gender, smoking habits, occupational exposure, and treatment for recent recurrence (stopped or ongoing). Like the previous study, BE sensitivity varied according to the staging and grading, with a higher sensitivity in higher stages $(52 \%, 100 \%, 100 \% \mathrm{for} \mathrm{Ta}, \mathrm{T} 1 \mathrm{and}$ CIS, respectively) and grades (40\% in low-grade, and $89 \%$ in high-grade tumors). NPV was high for the entire patient cohort (95\%). Notably, BE could exclude the presence of high-grade NMIBCs with a NPV of $99 \%$, and, conversely, it could detect the presence of high-grade NMIBCs with a sensitivity of $92 \%$. The AUC was 0.82 for all tumors (including low-grade Ta), and 0.94 when excluding low-grade recurrences.

Lozano et al. [14] recently updated the results of the previous study, with the addition of 382 patients, 304 of whom were recorded for results analysis (final cohort: 657 patients). Eighty patients were 'positive' as compared to the reference standard. The results were similar between the first and the second analyses (overall sensitivity: $62.5 \%$; sensitivity when excluding low-grade NMIBCs: $86 \%$; specificity: $86 \%$ ). NPV was $94 \%$. Notably, BE outperformed cytology in all categories (all-grade, low-grade, and high-grade NMIBCs). Moreover, in high-grade NMIBCs, BE showed a higher sensitivity than cystoscopy $(86 \%$ versus $73 \% ; p=0.113)$, although not significant. Conversely, BE sensitivity in the low-grade disease was significantly lower than cystoscopy ( $33 \%$ versus $94 \% ; p<0.0001$ ).

A secondary external independent analysis of the study by Witjes et al. [11] tested BE performance on 357 patients, with $49 / 357$ (13.7\%) intravesical disease recurrences. The study showed an overall sensitivity and specificity of $67 \%$ and $88 \%$, respectively. The sensitivity rose to $89 \%$ for the detection of high-grade NMIBCs. NPV was $94 \%$ for any-grade NMIBCs, and $99 \%$ for high-grade NMIBCs.

Considering univariable logistic regression analyses, a one-point increase in the EpiScore corresponded to a $4 \%$ increase in the risk of any-grade bladder cancer, and to an $8 \%$ increase in the risk of high-grade NMIBC. Using multivariable logistic regression analysis, a positive BE result was independently associated with the presence of any-grade (odds ratio, OR $=18.1,95 \%$ CI 8.66-40.2; $p<0.001)$ and high-grade NMIBC (OR $=78.3,95 \%$ CI 19.2-547; $p<0.001)$.

Finally, using explorative analysis, the performance of BE, evaluated by the AUC, was not affected by epidemiological features (age, gender, time from last recurrence, ongoing intravesical therapy, pathological stage and grade), and the addition of BE to these clinical variables significantly improved their AUC by $16 \%$ for the prediction of any-grade bladder cancer and $22 \%$ for the prediction of high-grade NMIBCs (BE AUC: $86 \%$ and $96 \%$ for any-grade and high-grade NMIBC, respectively).

During the study by Trenti et al. [12], BE was tested on 243 patients, with 215 evaluable results (28 test failures excluded from the final analysis). Sixty-nine (32\%) patients had an NMIBC recurrence. The overall BE sensitivity was $62 \%$, which rose to $83 \%$ for high-grade NMIBCs. The overall BE specificity was $86 \%$, and the NPV was $83 \%$. The diagnostic efficacy of BE was good, with an AUC of 0.785 . The lower accuracy and lower NPV of BE in this study was probably due to the higher prevalence of NMIBC recurrence.

A subsequent study by the same authors [13] compared BE to another Real-Time Polymerase Chain Reaction-Based urinary marker (Xpert Bladder Cancer Monitor [18]), which measures the level of five target messenger RNAs, upregulated in urine samples from patients with bladder cancer. The study enrolled 487 patients, 55 of whom had an invalid BE (total population: 432 patients). Twenty-one percent of the patients had an NMIBC recurrence. The test showed an overall sensitivity of $64 \%(66 \%$ for the Xpert Bladder Cancer Monitor test), which increased to 79\% in high-grade NMIBCs (57\% for low-grade NMIBCs). The overall specificity was $82 \%$ for the BE. The NPV was almost the same for both BE and Xpert Bladder Cancer Monitor (about 89\% for both). BE demonstrated a high diagnostic efficacy, with an AUC of $74 \%$ (95\% CI, 67.2-80.5\%).

A study by Righetto et al. [17] on 88 patients and $72 \mathrm{BE}$ results considered for the statistical analysis, showed a prevalence of recurrent NMIBC in $17.3 \%$ of the study population. The sensitivity of 
BE in all-grade NMIBCs was $76 \%$, which rose to $100 \%(95 \%$ CI, $75.5-100 \%)$ for high-grade NMIBCs. The overall specificity of the BE was $90 \%$, the NPV was $97 \%$ in all-grade NMIBCs, and $100 \%$ in high-grade NMIBCs.

Clinical data regarding the BE performance among the published studies are summarized in Table 1.

Table 1. Performance of Bladder Epicheck test among studies.

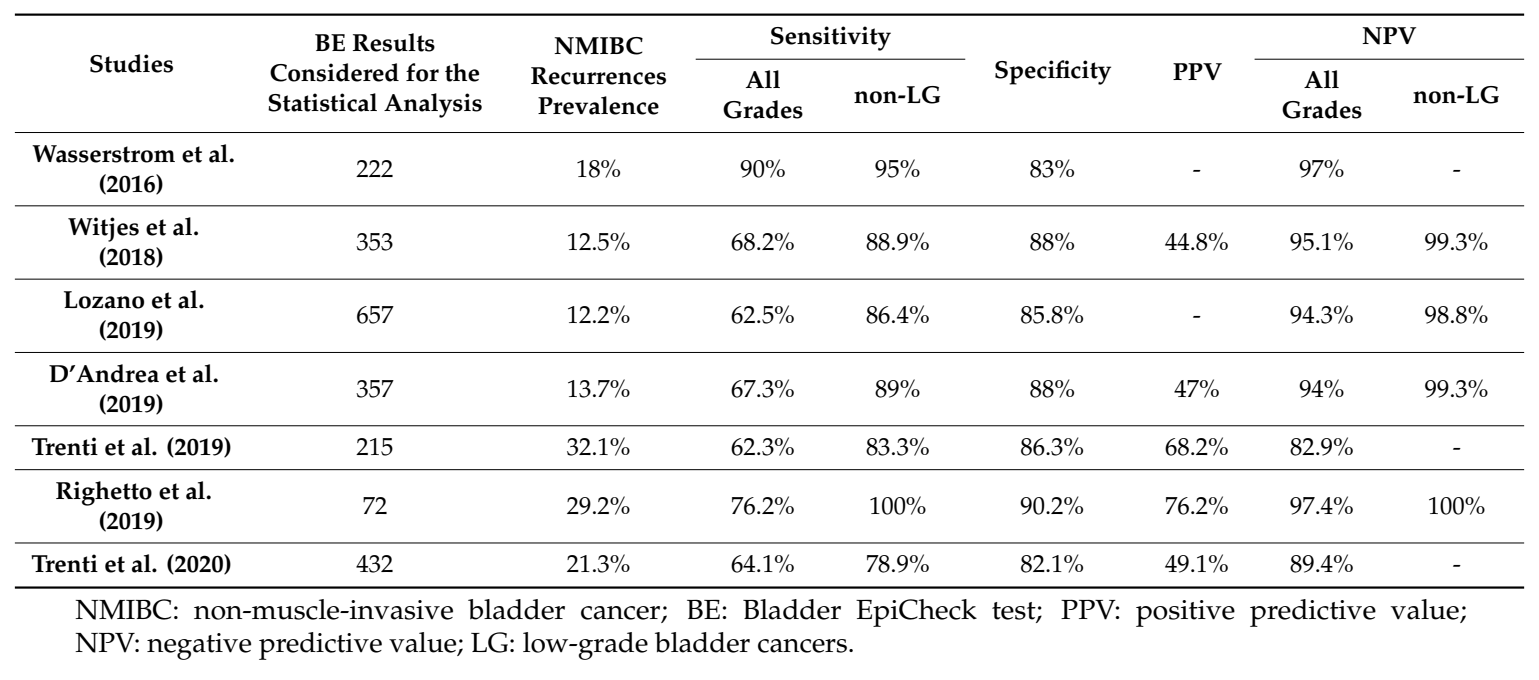

\subsection{Results: BE and Cytology}

Few studies have compared the sensitivity, PPV and NPV for both cytology and BE, and only one evaluated the diagnostic accuracy of the combination of the two tests [12].

Seen in all studies, the sensitivity of cytology was lower than the BE sensitivity (Table 2), with percentages ranging from $27 \%$ [13] to $38 \%$ maximum [15]. Particularly, the cytology sensitivity was markedly low in low-grade NMIBCs (0-13\% across studies), and increased, while remaining lower than BE sensitivity, in high-grade NMIBCs (50-67\% across studies). Notably, the NPV of the BE was higher than cytology NPV in all studies. Thus, the high sensitivity and NPV of BE could be used as an additional tool to improve the sensitivity of cytology.

The EpiScore, as a continuous variable, also could stratify differently according to different results of cytology. This data suggests that different cytological categories also carry a distinct methylation (thus, molecular) pattern.

Pierconti et al. [16] analyzed the EpiScore results of 374 patients with high-grade NMIBC (268 T1, 106 CIS) according to different cytological results (negative for high-grade urothelial carcinoma, NHGUC; atypical urothelial cells, AUC; suspicious for high-grade urothelial carcinoma, SHGUC; high-grade urothelial carcinoma, HGUC; low-grade urothelial neoplasia, LGUC; unsatisfactory/ non-diagnostic), finding that differences between cytological categories also could be based on molecular disparities. Actually, the EpiScore increased from NHGUC to HGUC; an EpiScore $<60$ correlated with NHGUC (OR $=3.9,95 \%$ CI 1.9-8.1, $p=0.0003$ ), while an EpiScore $\geq 60$ correlated with SHGUC $(\mathrm{OR}=3.8,95 \% \mathrm{CI} 1.6-8.9 ; p=0.0031)$ and HGUC (OR $=3.9,95 \%$ CI 1.6-9.5; $p=0.0027)$. Moreover, during a one year follow-up, the EpiScore had a sensitivity of $100 \%$, a specificity of $89.9 \%$, and a NPV of $100 \%$ in the NHGUC group while, in the HGUC group, the EpiScore reached a sensitivity of $98 \%$, a specificity of $100 \%$, and a NPV of $86 \%$.

A study by Trenti et al. [12] evaluated the possibility of combining BE and cytology results with the aim of increasing the diagnostic accuracy. The authors showed that the overall sensitivity of cytology $(33 \%)$ could significantly rise in combination with BE (67\% for the two tests combined). Particularly, the sensitivity for low-grade NMIBCs varied from $8 \%$ of cytology to $46 \%$ of $\mathrm{BE}$, while their combination yielded an overall sensitivity of $56 \%$. Conversely, the sensitivity for high-risk NMIBCs was $67 \%$ for 
cytology and $83 \%$ for $\mathrm{BE}$ alone, whereas the combination of the two tests obtained an overall sensitivity of $90 \%$.

The authors did not find a significant advantage in the combination of the two tests regarding PPV and NPV. The PPV was higher for cytology than for BE (92\% versus $68 \%$ ), while the NPV was similar for both $(75.8 \%$ for cytology, $82.9 \%$ for BE). The combination of the two tests failed to reach a significant improvement in PPV and NPV (overall PPV: 69\%, overall NPV: $84.5 \%$ ).

To conclude, the combination of both cytology and BE for the surveillance of patients with NMIBC could increase the sensitivity of the individual tests, especially in high-grade NMIBCs. Consequently, the combination of the two tests could potentially reduce the number and frequency of cystoscopies during follow-ups of patients with NMIBCs, especially for those with low-risk disease. However, we must consider that BE needs a dedicated and equipped laboratory to perform real time-PCR.

Table 2. Sensitivity, Specificity, PPV, and NPV of Cytology, Bladder EpiCheck, and the combination of the two tests between studies.

\begin{tabular}{|c|c|c|c|}
\hline \multirow{2}{*}{ Studies } & Cytology & EpiCheck & EpiCheck + Cytology \\
\hline & $\%,(95 \% \mathrm{CI})$ & $\%,(95 \%$ CI $)$ & $\%,(95 \% \mathrm{CI})$ \\
\hline \multicolumn{4}{|c|}{ Wasserstrom et al. (2016) } \\
\hline Sensitivity & $38 \%$ & $90 \%$ & \\
\hline Specificity & $96 \%$ & $83 \%$ & \\
\hline \multicolumn{4}{|l|}{ Trenti et al. (2019) } \\
\hline Sensitivity & $33.3(22.4-45.7)$ & $62.3(32.6-59.7)$ & $66.7(54.3-77.5)$ \\
\hline Low-grade NMIBC & $7.7(1.6-20.9)$ & $46.1(30.1-62.8)$ & $48.7(32.4-65.2)$ \\
\hline High-grade NMIBC & $66.7(47.2-82.7)$ & $83.3(65.3-94.4)$ & $90(73.5-97.9)$ \\
\hline Specificity & $98.6(95.1-99.8)$ & $86.3(79.6-91.4)$ & $85.6(78.8-90.9)$ \\
\hline PPV & $92.0(73.6-97.9)$ & $68.2(57.9-77.1)$ & $68.6(58.8-77.1)$ \\
\hline NPV & $75.8(72.6-78.7)$ & $78.6(72.5-83.9)$ & $84.5(79.4-84.7)$ \\
\hline \multicolumn{4}{|l|}{ Righetto et al. (2019) } \\
\hline Sensitivity & $35.7(12.8-64.8)$ & $76.2(52.8-91.8)$ & - \\
\hline Low-grade NMIBC & $0(0-60.2)$ & $37.5(85-75.5)$ & \\
\hline High-grade NMIBC & $50(18.7-81.3)$ & $100(75.3-100)$ & - \\
\hline Specificity & $96.8(83.3-99.9)$ & $90.2(78.6-96.7)$ & - \\
\hline Accuracy & $77.8 \%(62.9-88.8)$ & $86.1 \%(75.9-93.1)$ & \\
\hline Low-grade NMIBC & $85.7(69.7-95.2)$ & $83.0(71.0-91.6)$ & \\
\hline High-grade NMIBC & $85.4(70.8-94.4)$ & $92.2(82.7-96.7)$ & \\
\hline \multicolumn{4}{|l|}{ Trenti et al. (2020) } \\
\hline Sensitivity & $27.2(18.4-37.4)$ & $64.1(53.5-73.9)$ & - \\
\hline Low-grade NMIBC & $12.9(5.4-24.9)$ & $53.7(39.6-67.4)$ & \\
\hline High-grade NMIBC & $47.4(30.9-64.2)$ & $78.9(62.7-90.4)$ & \\
\hline Specificity & 98.8 (97.0-99.7) & $82.1(77.6-85.9)$ & - \\
\hline PPV & $86.2(69.0-94.6)$ & $49.2(42.4-55.9)$ & - \\
\hline NPV & $83.6(79.7-86.9)$ & $89.4(86.5-91.8)$ & - \\
\hline
\end{tabular}

PPV: positive predictive value; NPV: negative predictive value; CI: confidence interval.

\subsection{Results: Decision-Curve Analysis (DCA)}

The study by D'Andrea et al. [11] proposed two nomograms for the prediction of any-grade bladder cancer and high-risk bladder cancer during the follow-up of patients with NMIBC. The authors assessed the BE clinical utility and its impact on decision-making using decision-curve analysis (DCA), showing that current predictors of NMIBC recurrence (i.e., last stage and grade) performed no better than cystoscopy in every patient (which meant that all patients with NMIBC needed cystoscopy during their follow-up). Conversely, the use of BE for deciding whether to perform a cystoscopy or not during the follow-up of these patients offered a net benefit of BE relative to evaluating all patients with cystoscopy. This net benefit was maintained across threshold probabilities between 10-60\% for 
the presence of any-grade bladder cancer, and 5-55\% for high-grade bladder cancer. All decisions are currently based on clinical and epidemiological predictors, within these ranges. This data is fundamental during the decision-making process. BE could reduce the number of unnecessary cystoscopies and cytologies, without missing any cancer, in patients with a risk of recurrence $>10 \%$ for any-grade NMIBCs and $>5 \%$ for high-grade NMIBCs.

\subsection{BE and Other Bladder Tumor Markers}

Several molecular markers for both the diagnosis and follow-up of NMIBC are Food and Drug Administration approved. Particularly, for the follow-up of NMIBC, a non-invasive urine test with the best clinical utility should avoid unnecessary cystoscopies. Furthermore, such a urinary marker should have a high NPV—able to predict the absence of bladder cancer before cystoscopy.

Comparing BE and other markers used for the follow-up of patients with NMIBC [19], BE shows a higher sensitivity than a bladder tumor antigen stat test (BTA stat test; sensitivity range: $57-83 \%$ ), an ImmunoCyt (sensitivity range: $50-100 \%$ ), a fluorescence in situ hybridization (FISH-Urovysion; sensitivity: 70\%), and a nuclear matrix protein 22 (NMP 22) test (sensitivity range: $47-100 \%$ ).

However, there are two major points that play against BE as compared to other markers. First, $\mathrm{BE}$ requires a dedicated technician and a molecular laboratory to perform real time-PCR. Second, BE is not as cheap as the other tests.

\section{Conclusions}

BE has a high diagnostic accuracy and shows a high performance for surveillance of patients with NMIBC. Particularly, BE expresses the maximum diagnostic power in patients with low-grade bladder cancer. Regarding patients with a history of high-grade NMIBC, BE could be useful to increase the interval between cystoscopies, considering its very high NPV and high sensitivity.

BE showed, in several studies, a high NPV (see Table 2). It also showed that in positive cases it is able to select subsets of bladder cancer with negative cystoscopy and positive/negative cytology which seem to present DNA methylation changes. These 'false-positive' BE could represent a genetic high-risk population that could relapse in the near future and deserves close clinical attention. BE negative cases, on the other hand, could represent a population of high-risk bladder cancers with a more stable methylation pattern, which could present an inferior risk of progression.

Author Contributions: M.M. and M.R. contributed equally to study concept, design and drafting of the manuscript. All authors contributed to the critical revision of the manuscript for intellectual content. All authors have read and agreed to the published version of the manuscript.

Funding: This research received no external funding.

Conflicts of Interest: The authors declare no conflict of interest.

\section{References}

1. Babjuk, M.; Burger, M.; Compérat, E.M.; Gontero, P.; Mostafid, H.; Palou, J.; Van Rhijn, B.W.; Rouprêt, M.; Shariat, S.F.; Sylvester, R.; et al. European Association of Urology Guidelines on Non-muscle-invasive Bladder Cancer (TaT1 and Carcinoma In Situ)-2019 Update. Eur. Urol. 2019, 76, 639-657. [CrossRef] [PubMed]

2. Kassouf, W.; Traboulsi, S.L.; Schmitz-Dräger, B.J.; Palou, J.; Witjes, J.A.; Van Rhijn, B.W.; Grossman, H.B.; Kiemeney, L.A.; Goebell, P.J.; Kamat, A.M. Follow-up in non-muscle-invasive bladder cancer-International Bladder Cancer Network recommendations. Urol. Oncol. Semin. Orig. Investig. 2016, 34, 460-468. [CrossRef] [PubMed]

3. Leal, J.; Luengo-Fernandez, R.; Sullivan, R.; Witjes, J.A. Economic Burden of Bladder Cancer Across the European Union. Eur. Urol. 2016, 69, 438-447. [CrossRef] [PubMed]

4. Tilki, D.; Burger, M.; Dalbagni, G.; Grossman, H.B.; Hakenberg, O.W.; Palou, J.; Reich, O.; Rouprêt, M.; Shariat, S.F.; Zlotta, A.R. Urine Markers for Detection and Surveillance of Non-Muscle-Invasive Bladder Cancer. Eur. Urol. 2011, 60, 484-492. [CrossRef] [PubMed] 
5. Mancini, M.; Zazzara, M.; Zattoni, F. Stem Cells, Biomarkers and Genetic Profiling: Approaching Future Challenges in Urology. Urol. J. 2016, 83, 4-13. [CrossRef] [PubMed]

6. Kamoun, A.; De Reyniès, A.; Allory, Y.; Sjödahl, G.; Robertson, A.G.; Seiler, R.; Hoadley, K.A.; Groeneveld, C.S.; Al-Ahmadie, H.; Choi, W.; et al. A Consensus Molecular Classification of Muscle-invasive Bladder Cancer. Eur. Urol. 2020, 77, 420-433. [CrossRef] [PubMed]

7. Friedrich, M.G. Detection of Methylated Apoptosis-Associated Genes in Urine Sediments of Bladder Cancer Patients. Clin. Cancer Res. 2004, 10, 7457-7465. [CrossRef] [PubMed]

8. Beukers, W.; Kandimalla, R.; Masius, R.G.; Vermeij, M.; Kranse, R.; Van Leenders, G.J.; Zwarthoff, E.C. Stratification based on methylation of TBX2 and TBX3 into three molecular grades predicts progression in patients with pTa-bladder cancer. Mod. Pathol. 2014, 28, 515-522. [CrossRef] [PubMed]

9. Lotan, Y.; Shariat, S.F.; Schmitz-Dräger, B.J.; Sanchez-Carbayo, M.; Jankevicius, F.; Racioppi, M.; Minner, S.J.; Stöhr, B.; Bassi, P.F.; Grossman, H.B. Considerations on implementing diagnostic markers into clinical decision making in bladder cancer. Urol. Oncol. Semin. Orig. Investig. 2010, 28, 441-448. [CrossRef] [PubMed]

10. Witjes, J.A.; Morote, J.; Cornel, E.B.; Gakis, G.; Van Valenberg, F.J.P.; Lozano, F.; Sternberg, I.A.; Willemsen, E.; Hegemann, M.L.; Paitan, Y.; et al. Performance of the Bladder EpiCheck ${ }^{\mathrm{TM}}$ Methylation Test for Patients Under Surveillance for Non-muscle-invasive Bladder Cancer: Results of a Multicenter, Prospective, Blinded Clinical Trial. Eur. Urol. Oncol. 2018, 1, 307-313. [CrossRef] [PubMed]

11. D'Andrea, D.; Soria, F.; Zehetmayer, S.; Gust, K.M.; Korn, S.; Witjes, J.A.; Shariat, S.F. Diagnostic accuracy, clinical utility and influence on decision-making of a methylation urine biomarker test in the surveillance of non-muscle-invasive bladder cancer. BJU Int. 2019, 123, 959-967. [CrossRef] [PubMed]

12. Trenti, E.; D’Elia, C.; Mian, C.; Schwienbacher, C.; Hanspeter, E.; Pycha, A.; Kafka, M.; Degener, S.; Danuser, H.; Roth, S.; et al. Diagnostic predictive value of the Bladder EpiCheck test in the follow-up of patients with non-muscle-invasive bladder cancer. Cancer Cytopathol. 2019, 127, 465-469. [CrossRef] [PubMed]

13. Trenti, E.; Pycha, S.; Mian, C.; Schwienbacher, C.; Hanspeter, E.; Kafka, M.; Spedicato, G.A.; Vjaters, E.; Degener, S.; Pycha, A.; et al. Comparison of 2 new real-time polymerase chain reaction-based urinary markers in the follow-up of patients with non-muscle-invasive bladder cancer. Cancer Cytopathol. 2020, 128, 341-347. [CrossRef] [PubMed]

14. Palacio, F.L.; Morote, J.; Leibovitch, I.; Cornel, E.; Joyce, J.; Gakis, G.; Alvarez-Maestro, M.; Van Valenberg, H.; Sternberg, I.; Albers, L.; et al. Performance of bladder EpiCheck ${ }^{\mathrm{TM}}$ for NMIBC monitoring - updated results of a European multi-center study. Eur. Urol. Suppl. 2019, 18, e947-e949. [CrossRef]

15. Wasserstrom, A.; Frumkin, D.; Dotan, Z.; Bukin, E.; Gadish, T.; Hanuka, S.; Knirsh, R.; Darawsha, A.E.; Leibovitch, I. MP13-15 MOLECULAR URINE CYTOLOGY - BLADDER EPICHECK IS A NOVEL MOLECULAR DIAGNOSTIC TOOL FOR MONITORING OF BLADDER CANCER PATIENTS. J. Urol. 2016, 195, e140. [CrossRef]

16. Pierconti, F.; Martini, M.; Cenci, T.; Fiorentino, V.; Sacco, E.; Bientinesi, R.; Pugliese, D.; Iacovelli, R.; Schinzari, G.; LaRocca, L.M.; et al. Methylation study of the Paris system for reporting urinary (TPS) categories. J. Clin. Pathol. 2020. [CrossRef] [PubMed]

17. Righetto, M.; Zumerle, S.; Barcaro, F.; Saponaio, M.; Montopoli, M.; Ragazzi, E.; Pagano, F.; Zattoni, F.; Mancini, M. Genomic subtypes of bladder cancer with distinct methylation profiles could be better identified by the Bladder EpiCheck test as compared to cystoscopy/urine cytology. Eur. Urol. Suppl. 2019, 18, e3118. [CrossRef]

18. Pichler, R.; Fritz, J.; Tulchiner, G.; Klinglmair, G.; Soleiman, A.; Horninger, W.; Klocker, H.; Heidegger, I. Increased accuracy of a novel mRNA-based urine test for bladder cancer surveillance. BJU Int. 2017, 121, 29-37. [CrossRef] [PubMed]

19. Xylinas, E.; Kluth, L.A.; Rieken, M.; Karakiewicz, P.I.; Lotan, Y.; Shariat, S.F. Urine markers for detection and surveillance of bladder cancer. Urol. Oncol. Semin. Orig. Investig. 2014, 32, 222-229. [CrossRef] [PubMed]

(C) 2020 by the authors. Licensee MDPI, Basel, Switzerland. This article is an open access article distributed under the terms and conditions of the Creative Commons Attribution (CC BY) license (http://creativecommons.org/licenses/by/4.0/). 